\title{
A Conception of Pairwise Comparisons Model for Selection of Appropriate Body Surface Area Calculation Formula
}

\author{
Grzegorz Redlarski*†, Waldemar W. Koczkodaj ${ }^{\ddagger}$, Marek Krawczuk* ${ }^{*}$, Janusz Siebert ${ }^{\S}$, \\ Katarzyna E. Mrozik*, Aleksander Palkowski*, and Piotr M. Tojza* \\ ${ }^{*}$ Department of Mechatronics and High Voltage Engineering \\ Gdansk University of Technology, Gdansk, 80-233, Poland \\ Email: \{grzegorz.redlarski, marek.krawczuk\}@pg.gda.pl, \\ katmrozi@student.pg.gda.pl, \{aleksander.palkowski, piotr.tojza\}@pg.gda.pl \\ ${ }^{\dagger}$ Faculty of Technical Sciences \\ University of Warmia and Mazury, Olsztyn, 10-719, Poland \\ ‡Laurentian University, 935 Ramsey Lake Rd ON P3E 2C6 Sudbury, Canada \\ Email: wkoczkodaj@cs.laurentian.ca \\ $\S$ Department of Family Medicine \\ Medical University of Gdansk, Gdansk, 80-211, Poland \\ Email: kmr@gumed.edu.pl \\ IUniversity Center for Cardiology \\ Gdansk, Poland
}

\begin{abstract}
Body surface area (BSA) may be computed using a variety of formulas, but the computed BSA differs from real BSA values for particular subjects. This is presented in the paper by computing BSA values for selected subject and comparing them to the real BSA value obtained with the use of a 3D body scanner. The results show inequalities in the relevant BSA computing formulas. Hence, there is a need to determine a method that will allow to select the best formula for calculating BSA in a particular case. For this purpose, the pairwise comparisons (PC) method is suggested. This article presents a proposition of using consistency-driven PC, as well as the basic and most important aspects of using PC to determine the appropriate BSA calculation formula.
\end{abstract}

\section{INTRODUCTION}

During the last century, many different body surface area (BSA) formulas have been developed for use as the indicator of patient-focused health outcomes [1]. Commonly used BSA formulas had been reported as having different properties and accuracy in determination of real BSA values.

BSA is a parameter commonly used in medicine, mainly in oncology and burns treatment [1], [2]. It is crucial to determine the exact BSA value of the patient with minimal error using only the knowledge of patient's height and weight. Height and weight can be relatively easy to obtain especially when working under time pressure, which makes those factors suitable for medical examination. The existing methods used to compute BSA prove to be inaccurate and may cause ineffective chemotherapy or burns treatment. This article presents a proposition for evaluation and selection of appropriate BSA formula. The consistency-driven pairwise comparisons (PC) method is proposed to be used as an examination method for choosing a BSA formula for individuals based on a series of weighted parameters. Pairwise comparisons were also used in [3]-[5].

\section{BSA CALCULATION METHODS}

Since 1879 many BSA calculation formulas were developed, 25 of which are most common. To determine the BSA value these formulas use the patient's weight $W$ (in kilograms) and in most cases also patient's height $H$ (in centimetres). The formulas under question are listed in Table I.

\section{BSA CALCULATION ERRORS}

The early BSA calculation formulas were developed using different coating methods in order to obtain the patient's real BSA value. The accuracy of the methods depended not only on the type of mathematical approximation but also on the quality of the measuring methods. In the process of obtaining real BSA values the authors used subjects from a limited range of race, sex, and age, often generalizing the outcome BSA formula for an entire population.

In modern times, different methods of obtaining real BSA values were used. Still, when using the BSA calculation formulas the computed BSA values for the same patient vary in an extensive way. The results are not equal and differ one from another. This should not surprise when using old calculation formulas, but this regularity is true even for modern formulas.

To elucidate this phenomena a real BSA value was obtained from a female patient with the use of a body scanning device, specifically build for the purpose of scanning human bodies and obtaining high precision BSA. The scan was performed 
TABLE I

BODY SURFACE AREA FORMULAS CONSIDERED

\begin{tabular}{|c|c|c|}
\hline Authors & Formula & Reference \\
\hline Meeh (1879) & $0.1053 \cdot W^{2 / 3}$ & 6 \\
\hline DuBois \& DuBois (1916) & $0.007184 \cdot W^{0.425} \cdot H^{0.725}$ & 7 \\
\hline Faber \& Melcher (1921) & $0.00785 \cdot W^{0.425} \cdot H^{0.725}$ & 8 \\
\hline Takahira (1925) & $0.007246 \cdot W^{0.425} \cdot H^{0.725}$ & 9 \\
\hline Breitmann (1932) & $0.0087 \cdot(W+H)-0.26$ & 10 \\
\hline \multirow[t]{2}{*}{ Boyd (1935) } & 0.0003207 & \\
\hline & $\cdot(W \cdot 1000)^{0.7285-0.0188 \cdot \log _{10}(W \cdot 1000)} \cdot H^{0.3}$ & 11 \\
\hline Stevenson (1937) & $0.0128 \cdot W+0.0061 \cdot H-0.1529$ & 12 \\
\hline Sendroy \& Cecchini (1954) & $0.0097 \cdot(W+H)-0.545$ & 13 \\
\hline Banerjee \& Sen (1955) & $0.007466 \cdot W^{0.425} \cdot H^{0.725}$ & 14 \\
\hline Choi (1956) & $\begin{array}{l}\text { men: } 0.005902 \cdot W^{0.407} \cdot H^{0.776} \\
\text { women: } 0.008692 \cdot W^{0.442} \cdot H^{0.678}\end{array}$ & 15 \\
\hline Mehra (1958) & $0.01131 \cdot W^{0.4092} \cdot H^{0.6468}$ & 16 \\
\hline Banerjee \& Bhattacharya (1961) & $0.007 \cdot W^{0.425} \cdot H^{0.725}$ & 17 \\
\hline Fujimoto et al. (1968) & $0.008883 \cdot W^{0.444} \cdot H^{0.663}$ & 18 \\
\hline Gehan \& George (1970) & $0.0235 \cdot W^{0.51456} \cdot H^{0.42246}$ & 19 \\
\hline Haycock et al. (1978) & $0.024265 \cdot W^{0.5378} \cdot H^{0.3964}$ & 20 \\
\hline Mosteller (1987) & $\sqrt{W \cdot H / 3600}$ & 21 \\
\hline Mattar (1989) & $(W+H-60) / 100$ & 22 \\
\hline Nwoye (1989) & $0.001315 \cdot W^{0.262} \cdot H^{1.2139}$ & 23 \\
\hline Shuter \& Aslani (2000) & $0.00949 \cdot W^{0.441} \cdot H^{0.655}$ & 24 \\
\hline Livingston \& Lee (2001) & $0.1173 \cdot W^{0.6466}$ & 25 \\
\hline Tikuisis (2001) & $\begin{array}{l}\text { men: } 0.01281 \cdot W^{0.44} \cdot H^{0.6} \\
\text { women: } 0.01474 \cdot W^{0.47} \cdot H^{0.55}\end{array}$ & 26 \\
\hline Nwoye \& Al-Sheri (2003) & $0.02036 \cdot W^{0.427} \cdot H^{0.516}$ & 27 \\
\hline Yu, Lo, Chiou (2003) & $0.015925 \cdot(W \cdot H)^{0.5}$ & 28 \\
\hline Schlich (2010) & $\begin{array}{l}\text { men: } 0.000579479 \cdot W^{0.38} \cdot H^{1.24} \\
\text { women: } 0.000975482 \cdot W^{0.46} \cdot H^{1.08}\end{array}$ & 29 \\
\hline Yu, Lin, Yang (2010) & $0.00713989 \cdot W^{0.404} \cdot H^{0.7437}$ & 30 \\
\hline
\end{tabular}

with the use of Artec Eva 3D Scanner. The testes subject was a young 22-year-old female, of a body height of $171 \mathrm{~cm}$ and weight of $55.8 \mathrm{~kg}$ (Fig. 1a). After obtaining her real BSA value $\left(1.633 \mathrm{~m}^{2}\right)$, formulas presented in Table I were used to calculate individual BSA values. The results are shown in Fig. 1b. For most cases the results are greatly inconsistent with the real BSA value. The calculated BSA values span from $1.538 \mathrm{~m}^{2}$ (for the Meeh method) to $1.937 \mathrm{~m}^{2}$ (for the Nwoye method). Fig. 2 presents percentage values of errors between the real BSA value obtained through scanning and the formulas shown in Table I. The errors show that in most cases the formulas indicate BSA values lower than the real one. When taking into consideration the Meeh and Nwoye formulas, the maximum error that can be made in calculating BSA is $24.46 \%$. Therefore, it is important to develop the best method to select the right BSA calculation formula. For this purpose the PC model was selected.

To evaluate the above mentioned observation, a similar procedure concerning BSA calculation was performed in the case of 42 patients. The results are shown in Fig. 3. The patients used for this study were 20 to 28 year old, healthy Caucasians, both males and females. As it can be seen, the highest error values were obtained for the Nwoye method (12-15 percent in general) whereas the lowest error values characterizes the Yu, Lo and Chiou BSA calculation method (about 5 percent).

\section{DEFINITION OF WEIGHTS USED FOR BSA FORMULA SELECTION}

The PC method requires describing weights that are used in the selection process. They should be distinctive to the analysed process and represent the subjective assessment performed by a specialist (e.g., a physician) on the scale from 0 to 5 .

In the research process all scanned subjects can be divided to five groups based on their physique, age or medical history: normal, obese, after/during chemotherapy, elderly, and children. To each of these groups five factors are used in order to describe individual patients: degree of obesity, height to volume ratio, anthropological ancestry and race, type of body physique (athletic, deformed or similar), and degree of skin corrugation. The above mentioned classification and rating process is shown in Fig. 4.

\section{THE PC METHOD PRELIMINARIES}

The pioneer of PC is Condorcet [34]. He used PC in 1785 in the context of counting political ballots. In 1860, however, Fechner provided further, yet limited, psychometric information about this method. By way of refining the method, Thurstone [36] described the PC method as a statistical analysis and proposed a solution. In 1977 Saaty [37] introduced a hierarchy instrument for practical applications.

The PC method is outlined in Appendix A [31]-[33], [39]. It creates a matrix $A$ of values $a_{i j}$ of the $i$-th candidate (or alternative) compared with the $j$-th candidate. A scale $[1 / c, c]$ 
a

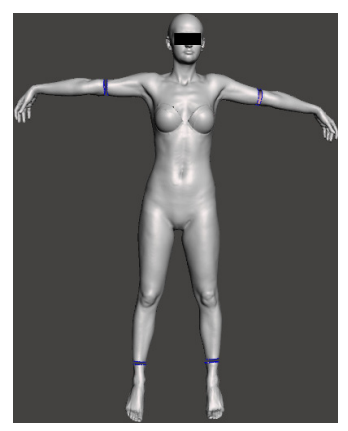

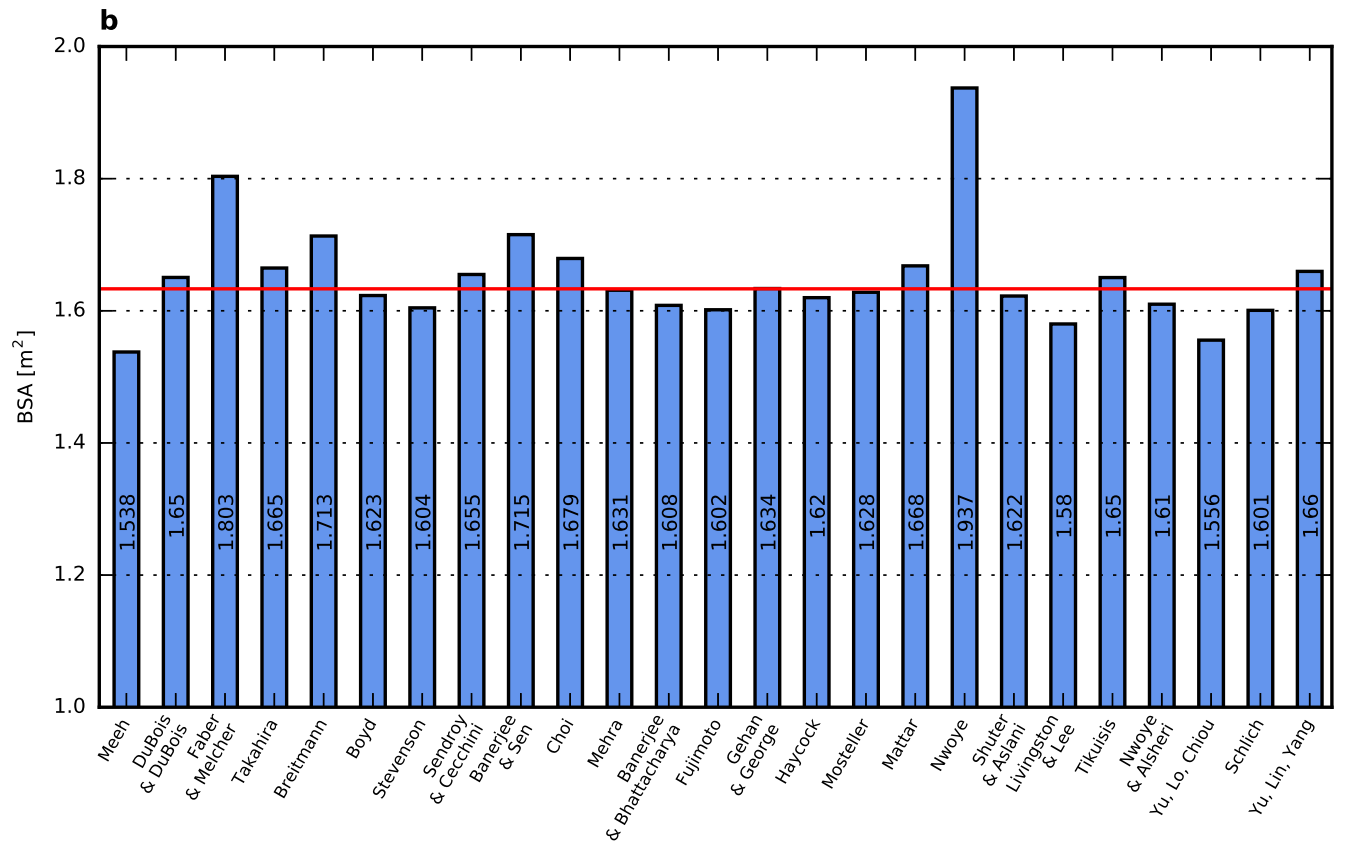

Fig. 1. BSA of a selected patient. a: 3D model of the patient. b: Calculated BSA values. The red line indicates the real BSA.

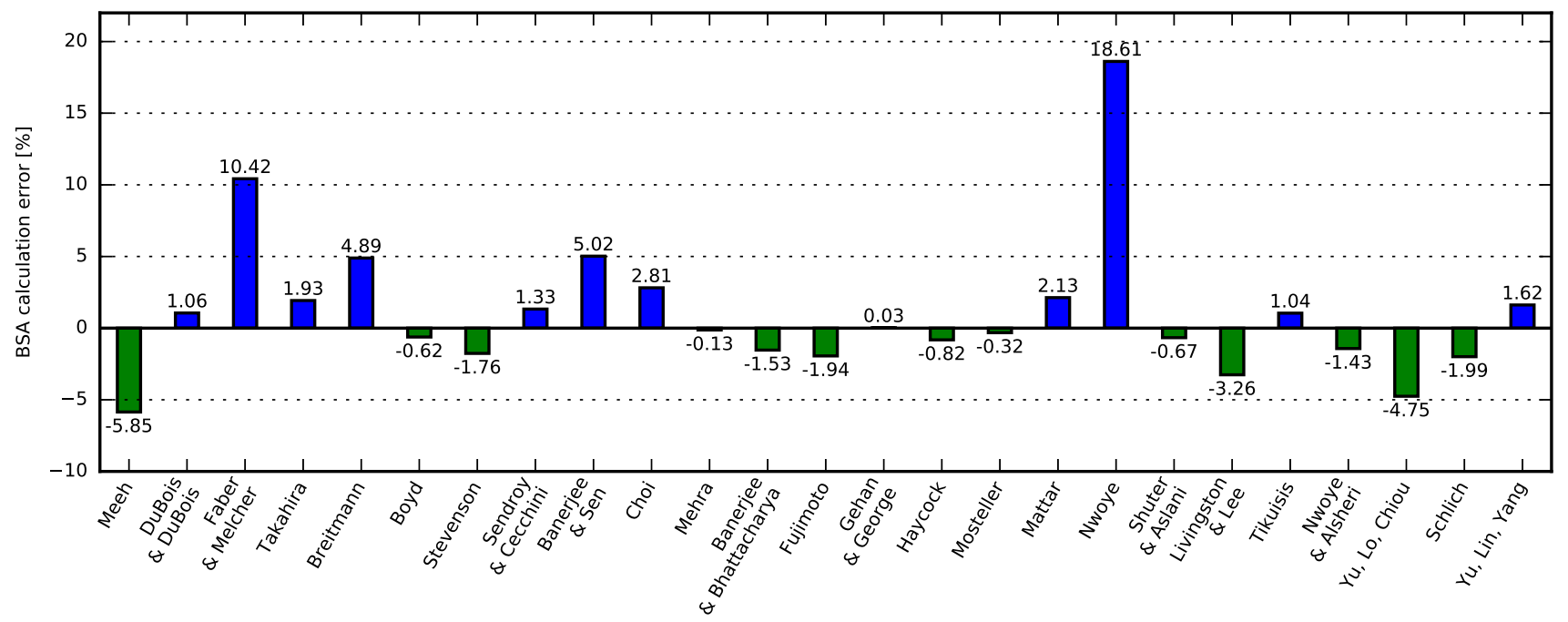

Fig. 2. Percentage error between the real BSA for a selected patient and BSA values calculated using the formulas shown in Table I.

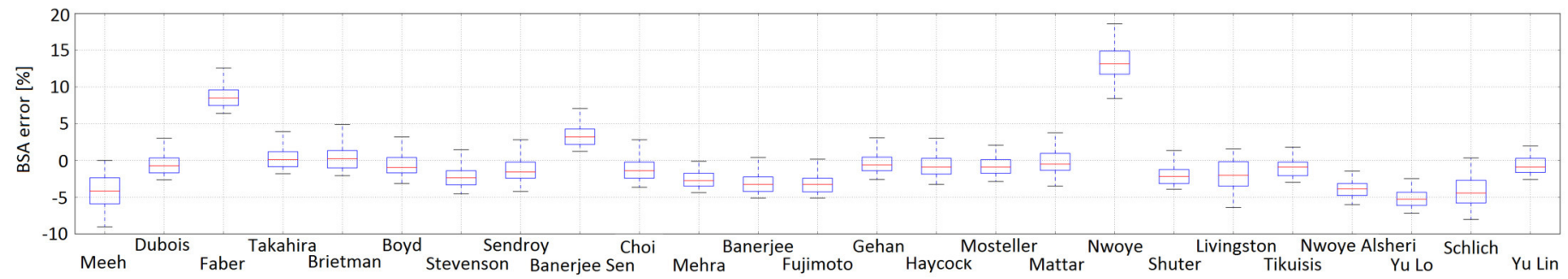

Fig. 3. Percentage error between the real BSA for a selected patient and BSA values calculated using the formulas shown in Table I for 42 patients. 


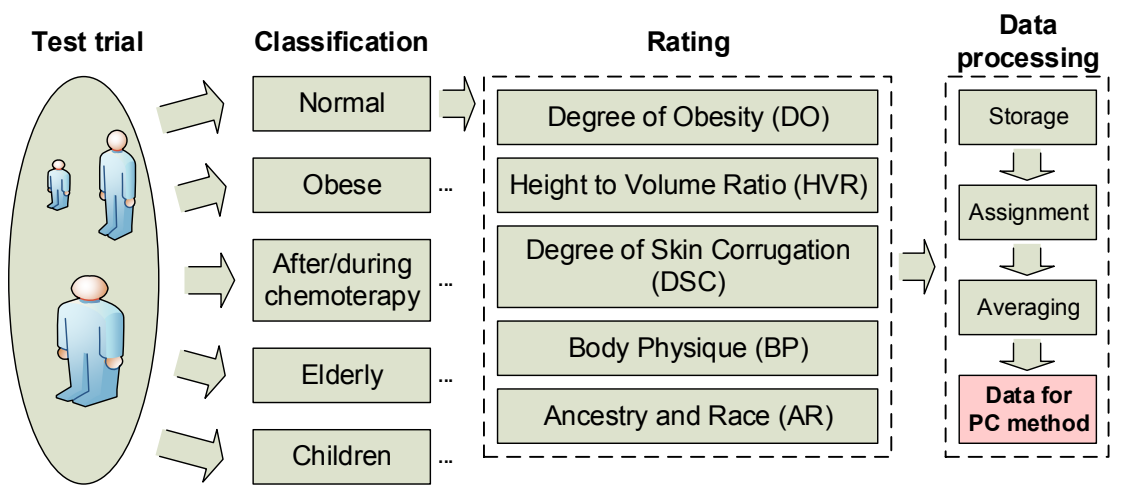

Fig. 4. Process of obtaining weights for factors used in the PC method.

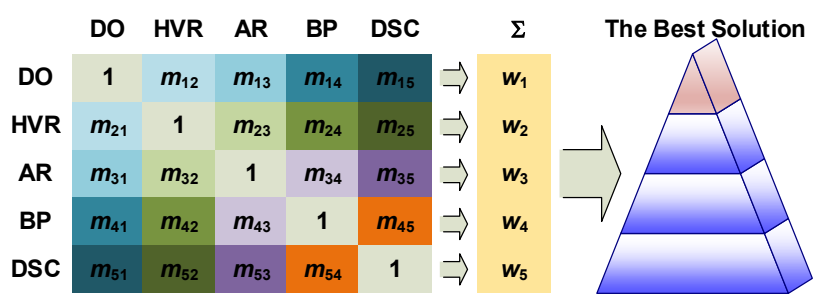

Fig. 5. The PC table of criteria and selection process.

is used for $i$ to $j$ comparisons where $c>1$ is a small real number ( 5 to 9 in most practical applications). It is usually assumed that all the values $a_{i j}$ on the main diagonal are equal to 1 .

Using a scale of 1 to 5 , the relative importance of each of the five groups are entered and objects are compared in the smallest subgroup. For example, degree of obesity and height to volume ratio are compared to each other in the subgroup and given 4 out of 5 (which can be changed for every clinical case to which this instrument is applied). In the case of inconsistency of the resulting matrix, the following formula [32] can be applied:

$$
\begin{array}{r}
i i=\min \left(\left|1-a_{i j} /\left(a_{i k} \cdot a_{k j}\right)\right|,\left|1-a_{i k} \cdot a_{k j} / a_{i j}\right|\right) \\
\text { for } i=1, j=2 \text {, and } k=3
\end{array}
$$

An example of a PC table of criteria and the selection process is presented in Fig. 5. The above presented factors are compared and relevant comparison values are assigned. Then the summed up weights of particular rows are compared with each other, thus providing an indicator for which of the tested solution is the best in the case.

As explained by Koczkodaj [32], [39], the weights $w_{1}$ to $w_{5}$ are computed as normalized geometric means of the matrix rows. The example is presented to illustrate the method, not the real instrument.

\section{CONCLUSION}

BSA is often a major factor in determining of the course of treatment. A series of formulas to simplify the process have been developed throughout the years. However, the choice of a particular formula is a difficult task. Therefore, there is a need to develop additional methods to help in the selection of an appropriate formula for individuals.

Although the PC method was originally used over 200 years ago, it has not been utilised to refine the properties of quality of life instruments. The method can strengthen the BSA calculation instrument by providing an additional layer of selection.

Evidently, not all objects on the BSA instrument are of equal importance. Appreciation of their relative differences adds to the measure's precision. The inconsistency analysis further strengthens the measure by bringing the most problematic but often crucial comparisons of the instrument items. A challenge to the multiple experts in this tool's development can be "averaging" their individual assessments in the assumed model. Clinical trials and statistical analysis need to follow the model enhancement.

The proposition presented in this paper show that it is possible to use the pairwise comparison in order to select a BSA calculation formula conformed to a specific situation. The enhancement may be a challenging undertaking for years to come. Refinement of the BSA may improve understanding of physiology as well as improve health care professional practices in their efforts to assess quality of life.

\section{ACKNOWLEDGMENT}

The research was supported by National Science Centre grant 2014/15/B/NZ7/01018.

\section{REFERENCES}

[1] Redlarski, G., Tojza, P.M.,Computer Supported Analysis of the Human Body Surface Area, International Journal of Innovative Computing, Information and Control. Vol 9, Issue 5, pp 1801-1818

[2] G. Redlarski, A. Palkowski, and M. Krawczuk, Body surface area formulae: an alarming ambiguity, Sci. Rep., vol. 6, p. 27966, Jun. 2016

[3] Mohammed Alqarni, Yassen Arabi, Tamar Kakiashvili, Mohammed Khedr, Waldemar W. Koczkodaj, J. Leszek, Artur Przelaskowski, K. Rutkowski: Improving the predictiveness of ICU medical scales by the method of pairwise comparisons. FedCSIS 2011: 11-17 
[4] Tamar Kakiashvili, Waldemar W. Koczkodaj, Phyllis Montgomery, Kalpdrum Passi, Ryszard Tadeusiewicz: Assessing the properties of the World Health Organization's Quality of Life Index. IMCSIT 2008: 151-154

[5] Waldemar W. Koczkodaj, Vova Babiy, Agnieszka D. Bogobowicz, Ryszard Janicki, Alan Wassyng: Selecting the best strategy in a software certification process. IMCSIT 2010: 53-58

[6] Meeh, K. Oberachenmessungen des menschlichen korpers. Zeitschrift fur Biologie 15, 425485 (1879).

[7] Du Bois, D. and Du Bois, E. F. A formula to estimate the approximate surface area if height and weight be known. Arch 117 Intern Med 17, 863871 (1916).

[8] Faber, H. K. and Melcher, M. S. A modication of the Du Bois heightweight formula for surface areas of newborn infants. 172 Experimental Biology and Medicine 19, 5354 (1921).

[9] Takahira, H. Metabolism of the Japanese. Imperial Government Institute of Nutrition. Report (The Institute, 1925).

[10] Breitmann, M. Eine vereinfachte Methodic der Korperoberachbestimmung. Zeitschrift fur Konstitutionslehre 17, $211 \quad 175214$ (1932).

[11] Boyd, E. Growth of Surface Area in Human Body. In Institute of Child Welfare Monograph Series, vol. 10, 5360177 (University of Minnesota Press, Minneapolis, 1935), 3rd edn.

[12] Stevenson, P. H. Height-weight-surface formula for the estimation of body surface area in Chinese subjects. The Chinese 179 Journal of Physiology 12, 327334 (1937).

[13] Sendroy, J. and Cecchini, L. P. Determination of Human Body Surface Area From Height and Weight. Journal of Applied 181 Physiology 7, 112 (1954).

[14] Banerjee, S. and Sen, R. Determination of the Surface Area of the Body of Indians. Journal of Applied Physiology 7, 183585588 (1955)

[15] Choi, W. R. The body surface area of Koreans. Ph.d. dissertation, Seoul National University (1956).

[16] Mehra, N. C. Body Surface Area of Indians. Journal of Applied Physiology 12, 3436 (1958)

[17] Banerjee, S. and Bhattacharya, A. K. Determination of body surface area in Indian Hindu children. Journal of Applied 187 Physiology 16, 969970 (1961).

[18] Fujimoto, S., Watanabe, T., Sakamoto, A., Yukawa, K. and Morimoto, K. Studies on the physical surface area of Japanese. 189 18. Calculation formulas in three stages over all ages. Japanese Journal of Hygiene 23 , 443450 (1968).

[19] Gehan, E. A. and George, S. L. Estimation of human body surface area from height and weight. Cancer chemotherapy 191 reports. Part 1 54, 225235 (1970).

[20] Haycock, G. B., Schwartz, G. J. and Wisotsky, D. H. Geometric method for measuring body surface area: A height-weight 193 formula validated in infants, children, and adults. The Journal of Pediatrics 93, 6266 (1978)

[21] Mosteller, R. D. Simplied calculation of body-surface area. The New England journal of medicine 317, 1098 (1987).

[22] Mattar, J. A. A Simple Calculation to Estimate Body Surface Area in Adults and Its Correlation with the Du Bois Formula. 196 Critical Care Medicine 17, 846853 (1989).

[23] Nwoye, L. O. Body Surface Area of Africans: A Study Based on Direct Measurements of Nigerian Males. Human 198 Biology 61, 439457 (1989).

[24] Shuter, B. and Aslani, A. Body surface area: Du Bois and Du Bois revisited. European Journal of Applied Physiology 82, 200250254 (2000).

[25] Livingston, E. H. and Lee, S. Body surface area prediction in normalweight and obese patients. American Journal of 202 Physiology - Endocrinology and Metabolism 281, E586E591 (2001).

[26] Tikuisis, P., Meunier, P. and Jubenville, C. Human body surface area measurement and prediction using three dimensional 204 body scans. European Journal of Applied Physiology 85, 264271 (2001).

[27] Nwoye, L. O. and Al-Shehri, M. A. A formula for the estimation of the body surface area of Saudi male adults. Saudi 206 Medical Journal 24 13411346 (2003).

[28] Yu, C.-Y., Lo, Y.-H. and Chiou, W.-K. The 3D scanner for measuring body surface area: a simplied calculation in the 119 Chinese adult Applied Ergonomics 34, 273278 (2003).

[29] Schlich, E., Schumm, M. and Schlich, M. 3D-Body-Scan als anthropometrisches Verfahren zur Bestimmung der spezis- 121 chen Korperoberache. Ernahrungs Umschau 4, 178183 (2010).
[30] Yu, C.-Y., Lin, C.-H. and Yang, Y.-H. Human body surface area database and estimation formula. Burns 36, 616629123 (2010).

[31] Kakiashvili, T., Kielan, K., Koczkodaj, W.W., Passi, K., Tadeusiewicz, R., Supporting the Asperger Syndrome Diagnostic Process by Selected AI Methods, proceedings of artificial intelligence studies. Vol. 4, pp21-27, 2007.

[32] Koczkodaj, W.W., A new definition of consistency of pairwise comparisons, Mathematical and computer modelling, (18)7, pp, 79-84, 1993.

[33] Koczkodaj, W.W., Herman, M.W., Orlowski, M. Using Consistencydriven Pairwise Comparisons in Knowledge-based Systems, International Conference on Information and Knowledge Management, 1997.

[34] Condorcet, M., The Essay on the Application of Analysis to the Probability of Majority Decisions, Paris: Imprimerie Royale, 1785.

[35] Fechner, G., Elemente der Psychophysik (1860, 2nd ed., 1889)

[36] Thurstone, L.L. (1927). A law of comparative judgement. Psychological Review, 34, 278-286.

[37] Saaty, T.L. (1977). A scaling method for priorities in hierarchical structures. Journal of Mathematical Psychology, 15, 234-281.

[38] McDowell. I., Measuring health : a guide to rating scales and questionnaires, 3rd ed., New York : Oxford University Press, 2006, 748 p.

[39] Koczkodaj, W.W., Redlarski, G., Szybowski, J., Wajch, E., Mikhailov, L., Soltys, M., Tamazian, G., Yuen, K.K.F., Important Facts and Observations about Pairwise Comparisons (the special issue edition), Fundamenta Informaticae 144, 1-17, 2016

\section{APPENDIX A}

\section{BASIC CONCEPTS OF PAIRWISE COMPARISONS}

An $n$ by $n$ pairwise comparisons matrix is defined as a square matrix $A=\left[a_{i j}\right]$ such that $a_{i j}>0$ for every $i, j=1, \ldots, n$. Each $a_{i j}$ expresses a relative preference of criterion (or stimulus) $s_{i}$ over criterion $s_{j}$ for $i, j=1, \ldots, n$ represented by numerical weights (positive real numbers) and $w_{i}$ and $w_{j}$ respectively. The quotients $a_{i j}=w_{i} / w_{j}$ form a pairwise comparisons matrix:

$$
A=\left[\begin{array}{cccc}
1 & a_{12} & \cdots & a_{1 n} \\
\frac{1}{a_{12}} & 1 & \cdots & a_{2 n} \\
\vdots & \vdots & \ddots & \vdots \\
\frac{1}{a_{1 n}} & \frac{1}{a_{2 n}} & \cdots & 1
\end{array}\right]
$$

A pairwise comparisons matrix $A$ is called reciprocal if $a_{i j}=1 / a_{j i}$ for every $i, j=1, \ldots, n$ (then automatically $a_{i i}=1$ for every $i=1, \ldots, n$ because they represent the relative ratio of a criterion against itself). A pairwise comparisons matrix $A$ is called consistent if $a_{i j} \cdot a_{j k}=a_{i k}$ holds for every $i, j, k=1, \ldots, n$ since $w_{i} / w_{j} \cdot w_{j} / w_{k}$ is expected to be equal to $w_{i} / w_{k}$. Although every consistent matrix is reciprocal, the converse is not generally true. In practice, comparing of $s_{i}$ to $s_{j}, s_{j}$ to $s_{k}$, and $s_{i}$ to $s_{k}$ often results in inconsistency amongst the assessments in addition to their inaccuracy; however, the inconsistency may be computed and used to improve the accuracy.

The first step in pairwise comparisons is to establish the relative preference of each combination of two criteria. A scale from 1 to 5 can be used to compare all criteria in pairs. Values from the interval $[1 / 5,1]$ reflect inverse relationships between criteria since $s_{i} / s_{j}=1 /\left(s_{j} / s_{i}\right)$. The consistency driven approach is based on the reasonable assumption that by finding the most inconsistent judgments, one can then reconsider one's own assessments. This in turn contributes to the improvement of judgmental accuracy. Consistency analysis is a dynamic process which is assisted by the software. 
The central point of the inference theory of the pairwise comparisons is Saaty's Theorem [37], which states that for every $n$ by $n$ consistent matrix $A=\left[a_{i j}\right]$ there exist positive real numbers $w_{1}, \ldots, w_{n}$ (weights corresponding to criteria $\left.s_{1}, . n . ., s\right)$ such that $a_{i j}=w_{i} / w_{j}$ for every $i, j=1, \ldots, n$. The weights $w_{i}$ are unique up to a multiplicative constant. Saaty (1977) also discovered that the eigenvector corresponding to the largest eigenvalue of $A$ provides weights $w_{i}$ which we wish to obtain from the set of preferences $a_{i j}$. This is not the only possible solution to the weight problem. In the past, a least squares solution was known, but it was far more computationally demanding than finding an eigenvector of a matrix with positive elements. Later, a method of row geometric means was proposed (Jensen, 1984), which is the simplest and most effective method of finding weights. A statistical experiment demonstrated that the accuracy, that is, the distance from the original matrix $A$ and the matrix $A N$ reconstructed from weights with elements $\left[a_{i j}\right]=\left[w_{i} / w_{j}\right]$, does not strongly depend on the method. There is, however, a strong relationship between the accuracy and consistency. Consistency analysis is the main focus of the consistency driven approach.

An important problem is how to begin the analysis. Assigning weights to all criteria (e.g., $A=18, B=27, C=20, D=$ $35)$ seems more natural than the above process. In fact it is a recommended practice to start with some initial values. The above values yield the ratios: $A / B=0.67, A / C=0.9$, $A / D=0.51, B / C=1.35, B / D=0.77, C / D=0.57$. Upon analysis, these may look somewhat suspicious because all of them round to 1 , which is of equal or unknown importance. This effect frequently arises in practice, and experts are tempted to change the ratios by increasing some of them and decreasing others (depending on knowledge of the case). The changes usually cause an increase of inconsistency which, in turn, can be handled by the analysis because it contributes to establishing more accurate and realistic weights. The pairwise comparisons method requires evaluation of all combinations of pairs of criteria, and can be more time consuming because the number of comparisons depends on $n^{2}$ (the square of the number of criteria). The complexity problem has been addressed and partly solved by the introduction of hierarchical structures [37]. Dividing criteria into smaller groups is a practical solution in cases in which the number of criteria is large.

\section{APPENDIX B}

\section{CONSISTENCY ANALYSIS}

Consistency analysis is critical to the approach presented here because the solution accuracy of not-so-inconsistent matrices strongly depends on the inconsistency. The consistency driven approach is, in brief, the next step in the development of pairwise comparisons.

The challenge to the pairwise comparisons method comes from a lack of consistency in the pairwise comparisons matrices which arises in practice. Given an $n$ by $n$ matrix $A$ that is not consistent, the theory attempts to provide a consistent $n$ by $n$ matrix $A N$ that differs from matrix $A$ "as little as possible". In particular, the geometric means method produces results similar to the eigenvector method (to high accuracy) for the ten million cases tested. There is, however, a strong relationship between accuracy and consistency.

Unlike the old eigenvalue based inconsistency, introduced in [37], the triad based inconsistency locates the most inconsistent triads [32]. This allows the user to reconsider the assessments included in the most inconsistent triad.

Readers might be curious, if not suspicious, about how one could arrive at values such as 1.30 or 1.50 as relative ratio judgments. In fact the values were initially different, but have been refined and the final weights have been computed by the consistency analysis. It is fair to say that making comparative judgments of rather intangible criteria (e.g., overall alteration and/or mineralization) results not only in imprecise knowledge, but also in inconsistency in our own judgments. The improvement of knowledge by controlling inconsistencies in the judgments of experts, that is, the consistency driven approach, is not only desirable but is essential.

In practice, inconsistent judgments are unavoidable when at least three factors are independently compared against each other. For example, let us look closely at the ratios of the four criteria $A, B, C$, and $D$ in Figure $C 1$. Suppose we estimate ratios $A / B$ as $2, B / C$ as 3 , and $A / C$ as 5 . Evidently something does not add up as $(A / B) @(B / C)=2 \cdot 3=6$ is not equal to 5 (that is $A / C$ ). With an inconsistency index of 0.17 , the above triad (with highlighted values of 2,5 , and 3 ) is the most inconsistent in the entire matrix (reciprocal values below the main diagonal are not shown in Figure $C 1$ ). A rash judgment may lead us to believe that $A / C$ should indeed be 6 , but we do not have any reason to reject the estimation of $B / C$ as 2.5 or $A / B$ as $5 / 3$. After correcting $B / C$ from 3 to 2.5 , which is an arbitrary decision usually based on additional knowledge gathering, the next most inconsistent triad is $(5,4,0.7)$ with an inconsistency index of 0.13 . An adjustment of 0.7 to 0.8 makes this triad fully consistent (5·0.8 is 4$)$, but another triad $(2.5,1.9,0.8)$ has an inconsistency of 0.05 . By changing 1.9 to 2 the entire table becomes fully consistent. The corrections for real data are done on the basis of professional experience and case knowledge by examining all three criteria involved.

An acceptable threshold of inconsistency is 0.33 because it means that one judgment is not more than two grades of the scale 1 to 5 away (an off-by-two error) from the remaining two judgments. There was no need to continue decreasing the inconsistency, as only its high value is harmful; a very small value may indicate that the artificial data were entered hastily without reconsideration of former assessments. 